\title{
THE WATERMARKING FOR 3D CAD DRAWING USING LINE, ARC, 3DFACE COMPONENTS
}

\author{
Ki-Ryong Kwon, ${ }^{1}$ Jae-Sik Sohn, ${ }^{2}$ Young Huh, ${ }^{3}$ Suk-Hwan Lee ${ }^{4}$ \\ ${ }^{l}$ Dept. of Electronics, Computer, and Telecommunication Engineering, Pukyong National University, \\ ${ }^{2}$ School of Electrical Engineering \& Computer Science, Kyungpook National University, \\ Korea Electrotechnology Research Institute, \\ ${ }^{4}$ Dept. of Information Security, TongMyong University
}

\begin{abstract}
Currently there has been much interested in developing the watermarking for 3D graphic data of mesh model or NURBS. However, the watermarking technique based on 3D CAD drawing leaves something to be desired. This paper proposes a watermarking technique for $3 \mathrm{D}$ design drawing using the components of Line, 3DFACE and ARC based on vertex that prevent the infringement of copyright from unlawfulness reproductions and distribution. By experimental result, we confirmed the invisibility of embedded watermarks as well as the robustness in geometrical attacks and file conversion to DWG, DXF, DWT and DWS.
\end{abstract}

\section{INTRODUCTION}

With the rapid increase of the multimedia information in information-communication technology, the intellectual property and copyright protection has been made at issue. Generally there are two technologies for the intellectual property and copyright protection; cryptography and watermarking. The cryptography technology cut off the access of the unauthorized person after the multimedia information is encrypted. However, it cannot prevent the unlawful action of an authorized person and cannot solve the problem that some copyright owners assert their ownerships for one content. To solve the problems of the cryptography, there have been much researched in watermarking technology, which is the end-step in information security and protects the copyright of owner by embedding the watermark into the multimedia information.

A lot of research has been carried out to protect the copyright protection of image, video, and audio [1]. Recently, 3D graphic models, such as VRML, MPEG-4, and 3D geometrical CAD, have become very popular leading the development of 3D watermarking algorithms to protect the copyright of 3D graphic models with the extending technique of the image watermarking. The watermarking for 3D polygonal model and 2D Vector data has begun to be studied. Ohbuchi et al. presented the watermarking for $3 \mathrm{D}$ polygonal model through geometric and topological modification [2]. Mao et al. presented the watermarking for 3D geometric model through the triangle subdivision [3]. Beneden et al. also presented an algorithm that adds a watermark by modifying the normal distribution of the model geometry [4]. Kanai et al. presented the watermarking for $3 \mathrm{D}$ polygons using the multiresolution wavelet decomposition [5]. Ohbuchi et al. [6] presented Vector digital map watermarking using rectangles that have vertices of fixed quantity by quad-tree way. Many design drawings in the industrial filed have been designed by $3 \mathrm{D}$ CAD tools. However, because of the unlawful reproduction of the architectural drawings, the construction industry has been financially damaged.

This paper proposed the watermarking for $3 \mathrm{D}$ CAD drawing based on $3 \mathrm{D}$ vector data, which is a public watermarking using Line, Arc, and 3DFACE components in $3 \mathrm{D}$ CAD drawing. The watermark is embedded into the length of Line component in the embedding primitives, the circular radius of Arc component, and the length ratio of two sides in 3DFACE component. Thus, according to the distribution of three components in 3D CAD drawing, the embedding component can be determined. The results of experiment verify that the proposed algorithm is imperceptible and robust against file format conversion, move, scaling, rotation, component cropping, and layer cropping.

\section{THE PROPOSED 3D CAD WATERMARKING}

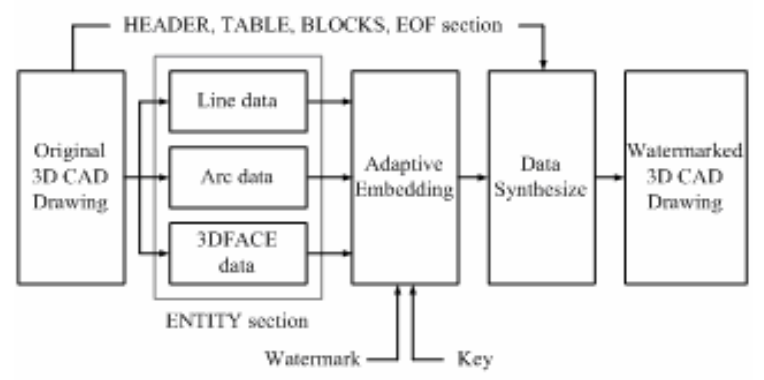

Fig. 1. Proposed 3D CAD watermark embedding system.

\subsection{D CAD drawing}


The basic system of 3D CAD drawings consists of HEADER, TABLE, BLOCK, ENTITY, EOF sections. The shape of drawings is designed with the basic component of Line, Arc, Circle, and 3DFACE based on vector data in ENTITY section. 3D CAD drawings can be attacked intentionally or non-intentionally by CAD tools. The general attacks in CAD tools are followed as; 1) File Format Conversion: CAD drawings are easily converted to the format in AutoCAD such as DXF, DWG, DWT, and DWS. 2) Geometrical attack: There are translation, scaling, rotation, and cropping. 3) Layer cutting: The drawings in AutoCAD are composed of several layers. Users are able to cut some layers illegally. The watermark in $\mathrm{CAD}$ drawings must be robust against the above attacks.

\subsection{Watermark Embedding}

The proposed watermarking embeds the watermark into the components of Line, Arc, and 3DFACE respectively according to the structure of 3D CAD drawings, as shown in Fig. 1. Three components are obtained from ENTITY section of CAD data. The embedding primitives are the length in Line, the radius of curvature in Arc, and the length ratio of two sides in 3DFACE. They are stored to extract the watermark as the key.

\subsubsection{Line component}

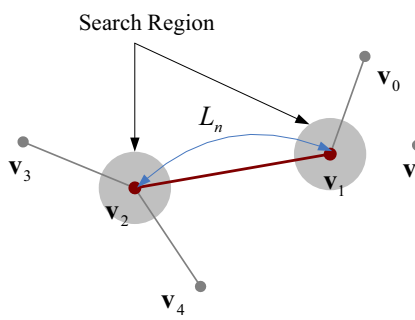

(a)

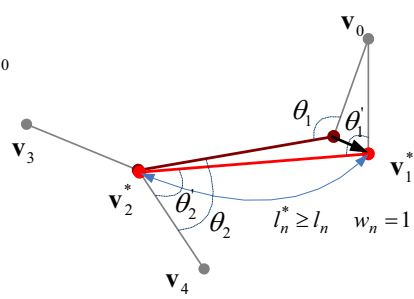

(b)
Fig. 2. (a) An embedding primitive in Line components and (b) embedding the watermark into a embedding primitive.

A Line component consists of the start point $\mathbf{v}_{s}=\left(x_{s}, y_{s}, z_{s}\right)$ and the end point $\mathbf{v}_{e}=\left(x_{e}, y_{e}, z_{e}\right)$. Each point may be connected to the neighborhood points. If a point is varied by the watermark, the neighborhood points will be varied together. The set of embedding primitive that consists of an arbitrary point and the neighborhood points connected to a point is gathered to embed the watermark. A bit of binary watermark is embedded into one point of a center line in an embedding primitive. Thus, a $n$th watermark bit $w_{n}$ is embedded into the length $l_{n}$ of center line as follows; if $w_{n}=1$, then $l_{n} \geq \bar{l}_{n}$. Otherwise, $l_{n}<\bar{l}_{n}$. $\bar{l}_{n}$ is an average length of the connected Lines. To change the length of line according to the watermark, the coordinate of the point must be changed imperceptibly considering the neighborhood points. Fig. 2 (a) shows a center line $L_{n}=\left\{\mathbf{v}_{1} \mathbf{v}_{2}\right\}$ and 3 neighborhood lines in an embedding primitive. $\mathbf{v}_{0}$ is connected to $\mathbf{v}_{1}$ and $\mathbf{v}_{3}, \mathbf{v}_{4}$ are connected to $\mathbf{v}_{2}$. The search regions of $\mathbf{v}_{1}, \mathbf{v}_{2}$ which can be changed invisibly are determined respectively to be below the coordinate values of the connected points. Thus, the search region of $\mathbf{v}=(x, y, z)$ is $[\mathbf{v}-\Delta \mathbf{v}, \mathbf{v}+\Delta \mathbf{v}]$,

$\Delta \mathbf{v}=0.5 \times \min \left|t-\mathbf{v}_{k}^{t}\right|_{\mathbf{v}_{t} \in C(\mathbf{v}), t \in\{x, y, z\}}$ where $C(\mathbf{v})$ represents the points that are connected to $\mathbf{v}$. Two points $\mathbf{v}_{1}, \mathbf{v}_{2}$ are changed to $\mathbf{v}_{1}^{\prime}=\mathbf{v}_{1} \pm \alpha, \mathbf{v}_{2}^{\prime}=\mathbf{v}_{2} \pm \alpha$ alternatively within the search regions until satisfies the condition as follows; $\min _{\theta_{1 k} \in \Theta_{1}}\left|\theta_{1 k}-\theta_{1 k}^{\prime}\right|<\varepsilon, \min _{\theta_{2 k} \in \Theta_{2}}\left|\theta_{2 k}-\theta_{2 k}^{\prime}\right|<\varepsilon$ where $\theta_{1 k}=\cos ^{-1}\left(\frac{\overrightarrow{\mathbf{v}_{1} \cdot \mathbf{v}_{2}} \cdot \overrightarrow{\mathbf{v}_{1} \cdot \mathbf{v}_{1 k}}}{\left\|\overrightarrow{\mathbf{v}_{1} \cdot \mathbf{v}_{2}}\right\|\|\| \overrightarrow{\mathbf{v}_{1} \cdot \mathbf{v}_{1 k}} \|}, \theta_{2 k}=\cos ^{-1}\left(\frac{\overrightarrow{\mathbf{v}_{1} \cdot \mathbf{v}_{2}} \cdot \overrightarrow{\mathbf{v}_{2} \cdot \mathbf{v}_{2 k}}}{\left\|\overrightarrow{\mathbf{v}_{1} \cdot \mathbf{v}_{2}}\right\|\left\|\overrightarrow{\mathbf{v}_{2} \cdot \mathbf{v}_{2 k}}\right\|}\right)\right.$ and $\mathbf{v}_{1 k} \in C\left(\mathbf{v}_{1}\right), \mathbf{v}_{2 k} \in C\left(\mathbf{v}_{2}\right)$.

\subsubsection{Arc component}

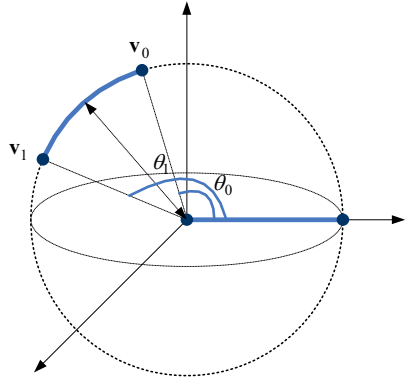

(a)

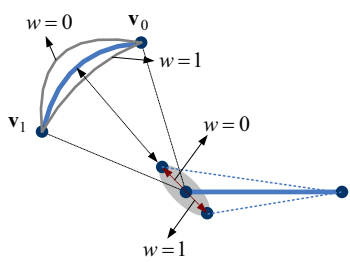

(b)
Fig. 3. (a) The structure of an Arc component and (b) the watermark embedding according to the center point $\mathbf{C}$.

An Arc component consists of two points $\mathbf{v}_{0}, \mathbf{v}_{1}$, a circle center point $\mathbf{C}$, a circle radius $R$, a standard point of angle $\mathbf{P}$, two angles $\theta_{0}, \theta_{1}$ between points and $\mathbf{P}$ as shown in Fig. 3 (a). The watermark bit is embedded into a circle radius $\mathbf{R}$ in the randomly selected Arc component. If a watermark bit $w_{n}$ is $0, \quad \mathbf{C}$ is moved forward to $\mathbf{m}=\left(\mathbf{v}_{0}+\mathbf{v}_{1}\right) / 2$. Otherwise, $\mathbf{C}$ is moved backward to $\mathbf{m}$. $\mathbf{C}$ must be moved within the limit region that the differential ratio of the curvature $\Delta \kappa=\kappa-\kappa^{\prime}=1 / R-1 / R^{\prime}$ is below $\varepsilon . R^{\prime}$ is the circle radius of Arc with $C^{\prime}$ which is moved according to the watermark bit.

\subsubsection{DFACE component}

3DFACE represents 3D polygon surface that can be in general use for the surface modeling in 3D CAD drawing. In this paper, 3DFACE components over quadrilateral are used for the watermark embedding. In the randomly 
selected a 3DFACE component, the set of the distance between two points is obtained; $L=\left\{l_{0}, l_{1}, \cdots l_{n}\right\}$, $l_{i}=\left|\mathbf{v}_{i} \mathbf{v}_{i+1}\right|$. An arbitrary distance pair $\left\{l_{i}, l_{j}\right\}, l_{i}, l_{j} \in L$, $i<j,\left|l_{i}-l_{j}\right|<\Delta$ without the shared point is randomly selected where $l_{i}=\left|\mathbf{v}_{i} \mathbf{v}_{i+1}\right|$ is a reference distance, $l_{j}=\left|\mathbf{v}_{j} \mathbf{v}_{j+1}\right|$ is the changeable distance.

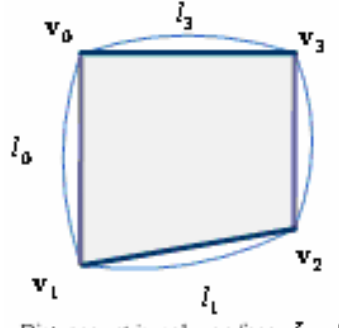

Distance set in polygon face $L=\left\{l_{0}, l_{1}, l_{2}, l_{1}\right\}$ Embedding primitives $\left\{l_{0}, l_{1}\right\},\left\{l_{2}, l_{3}\right\}$

(a)

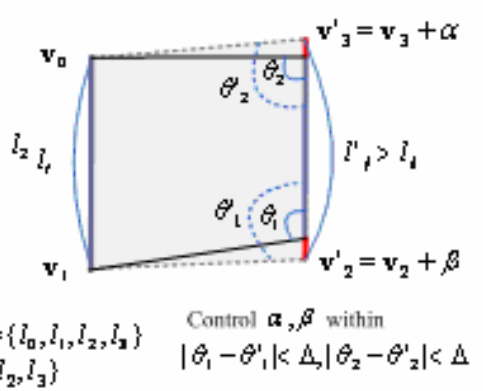

(b)
Fig. 4. (a) The structure of 3DFACE component with 4 faces and (b) the embedding using the ratio of two distances when $w=0$.

The watermark bit $w$ is embedded into the ratio $\alpha=l_{i} / l_{j}$ of distance pair; $\alpha \geq 1$ if $w=1$ and $\alpha<1$ otherwise. To change the distance ratio according to the watermark bit, two points $\mathbf{v}_{j}, \mathbf{v}_{j+1}$ in the changeable distance $l_{j}$ must be changed to be parallel the reference distance $l_{i}$ within the invisible range as shown in Fig. 4. The invisible ranges of two points are $\left|\theta_{j}-\theta_{j}^{\prime}\right|<\Delta$ and $\left|\theta_{j+1}-\theta_{j+1}^{\prime}\right|<\Delta$ respectively. $\theta_{j+k}$ and $\theta_{j+k}^{\prime}$ are the angles of two lines with the original intersection point, $\mathbf{v}_{j+k}$, or the changed point $\mathbf{v}_{j+k}^{\prime}, k=0,1 ; \theta_{j+k}=\cos ^{-1}\left(\frac{\overrightarrow{\mathbf{v}_{j+k} \mathbf{v}_{j+k+1}} \cdot \overrightarrow{\mathbf{v}_{j+k} \mathbf{v}_{j+k-1}}}{\left\|\overrightarrow{\mathbf{v}_{j+k} \mathbf{v}_{j+k-1}}\right\| \| \overrightarrow{\mathbf{v}_{j+k} \mathbf{v}_{j+k-1} \|}}\right)$.

\subsection{Watermark Extracting}

The watermark is extracted from the watermarked drawing using two points of the embedded Line component, a circle radius of the embedded Arc component, and the embedding primitive of the embedded 3DFACE component on the same as the embedding algorithm. But to extract the watermark in the watermarked drawing scaled to an arbitrary factor, the re-scaling process is performed by using $\overline{l^{*}}, \overline{R^{*}}, \overline{A^{*}}$, which are an average length, circle radius, area of all the embedded Line, Arc, 3DFACE. All components are re-scaled to dilated or shrinked until all ratios $\overline{l^{*}} / \overline{l^{\prime}}, \overline{R^{*}} / \overline{R^{\prime}} \overline{A^{*}} / \overline{A^{\prime}}$ are $1 . \overline{l^{\prime}}, \overline{R^{\prime}}, \overline{A^{\prime}}$ are an average length, circle radius, area of Line, Arc, 3DFACE in the attacked drawing. It takes long time to re-scale closely to original scale factor.

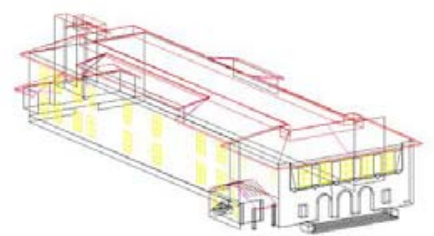

(a)

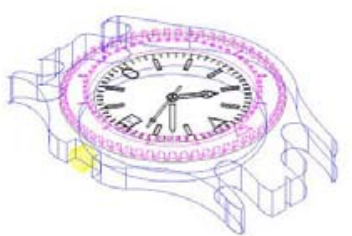

(b)

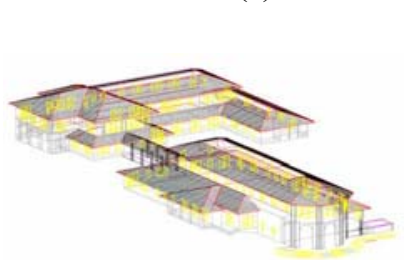

(c)

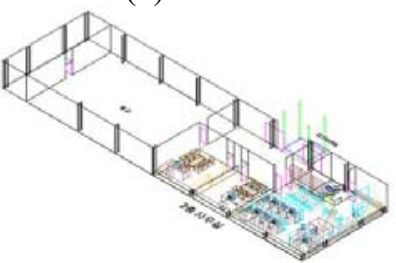

(d)
Fig. 5. 3D CAD drawings in AutoCAD; (a) Campus1, (b) Watch, (c) Campus2, and (d) Office.

\section{EXPERIMENTAL RESULTS}

To evaluate the performance of the proposed watermarking, we used 3D CAD drawings designed by AutoCAD 2002 software; Campus, Watch, and Office drawings as shown in Fig. 5. The watermark was used as bit stream generated by a Gaussian random sequence. The length of watermark can be determined by the component distribution of the 3D CAD drawing. There are a number of Line components in Campus1 drawing, Arc components in Watch drawing, 3DFACE components in Campus2, and Line, 3DFACE components in Office as shown in Fig. 5. Among these components in each drawing, we selected 500 components for the embedding primitives. The 3D CAD drawings that are watermarked by the proposed algorithm are shown in Fig. 6. In this figure, a subjective evaluation was used to verify that the watermark was invisible. For the objective evaluation for visibility, we used SNR of each embedding components, which are SNR of points in Line, 3DFACE and radius in Arc. Table 1 shows that SNRs of the watermarked drawings are about $39.89-42.50 \mathrm{~dB}$ according to the number of components. These values verify the good quality.

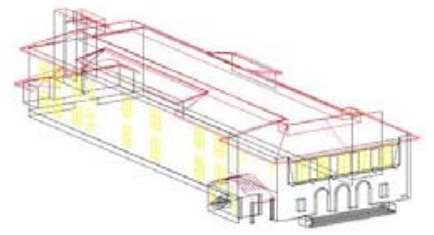

(a)

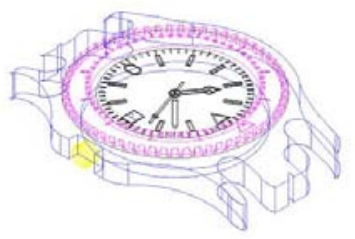

(b)
Fig. 6. The watermarked (a) Campus1 and (b) Watch drawings. 
Table 1. SNR of the watermarked 3D CAD drawings.

\begin{tabular}{c|c|c|c|c}
\hline Test drawing & Campus & Watch & Campus & Floor \\
\hline $\begin{array}{c}\text { Embedding } \\
\text { component } \\
\text { (Number) }\end{array}$ & $\begin{array}{c}\text { Line } \\
(1,130)\end{array}$ & $\begin{array}{c}\text { Arc } \\
(1,770)\end{array}$ & $\begin{array}{c}\text { 3DFACE } \\
(551)\end{array}$ & $\begin{array}{c}\text { Line(8,738) } \\
\text { 3DFACE } \\
(3,927)\end{array}$ \\
\hline SNR & $40.12 \mathrm{~dB}$ & $41.05 \mathrm{~dB}$ & $39.89 \mathrm{~dB}$ & $\begin{array}{c}42.50 \mathrm{~dB}, \\
41.33 \mathrm{~dB}\end{array}$ \\
\hline
\end{tabular}

To evaluate the robustness, the watermarked drawings were attacked by file format conversion, RST (translation, scaling, rotation), cropping, and layer cutting. In file format conversion, the watermarked drawings were converted to DXF, DWG, DWT, and DWS by using AutoCAD. But, the watermark can be extracted without bit error in any file format. For geometrical attacks, the watermarked drawings were translated to arbitrary point, dilated or shrinked to arbitrary scaling factor, rotated to arbitrary angle, and cropped to $30 \%$ of the embedding components. Since the proposed algorithm embeds the watermark into the length in Line, the radius of curvature in Arc, and the length ratio of two sides in 3DFACE, it has not effect on translation and rotation. In scaled drawing, the watermark has to be extracted after performing re-scaling process. Table 2 verifies that all watermark bits can be extracted without bit error. But in cropping, the bit error occurred about 0.08$0.22 \%$ according to the cropping percentage of components Furthermore, we cut an arbitrary layer in the watermarked drawings that similar as the cropping of all components in a layer. In this case, the bit error occurred about 0.15-0.28 that effects on the number of the embedding components in a layer. Since Floor drawings were embedded the watermark into two components, BER is an average of BERs in two components. The above results verified that the watermark still alive above $78 \%$ in any attacks.

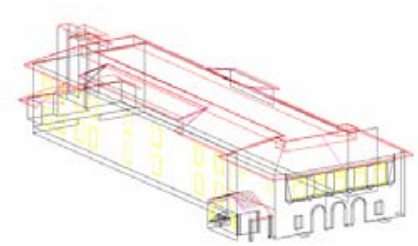

(a)

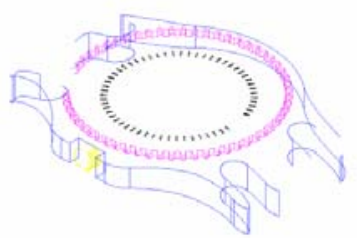

(c)

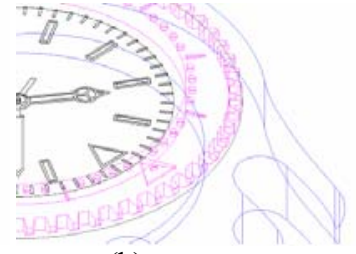

(b)

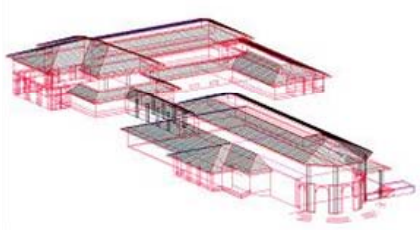

(d)
Fig. 7. (a) A cropped Campus 1 to $30 \%$ of Line components, (b) a scaled Watch to 3 times, and (c) Watch, (d) Campus2 with layer cutting.
Table 2. BER of the extracted watermark.

\begin{tabular}{c|c|c|c|c}
\hline Attack Test drawing & Campus & Watch & Campus & Floor \\
\hline $\begin{array}{c}\text { Format conversion } \\
\text { (DXF,DWG,DWT,DWS) }\end{array}$ & - & - & - & - \\
\hline RST & - & - & - & - \\
\hline $30 \%$ cropping & 0.13 & 0.08 & 0.22 & 0.03 \\
\hline Layer cutting & 0.24 & 0.15 & 0.28 & 0.07 \\
\hline
\end{tabular}

\section{CONCLUSIONS}

This paper presented a watermarking for 3D CAD drawings using Line, Arc, and 3DFACE components. The embedding components can be selected randomly or by the component distribution in drawing. The watermark is embedded into the length in Line component, the radius of curvature in Arc, and the length ratio of two sides in 3DFACE. Experimental results verified that the proposed watermarking has the robustness against Format conversion, RST, cropping, and layer cutting as well as the invisibility in a view of component SNR.

\section{ACKNOLWEDGEMENT}

This work was supported by the Korea Research Foundation Grant funded by the Korean Government (MOEHRD)" (KRF-2005-042-D00225), in which main calculations were performed by using the supercomputing resource of the Korea Institute of Science and Technology Information (KISTI) and by grant No. R01-2006-00010260-0 from the Basic Research Program of the Korea Science \& Engineering Foundation.

\section{REFERENCES}

[1] I.J.Cox, J.Kilian, T.Leighton, and T.Shamoon, "Secure Spread Spectrum Watermarking for Multimedia," NEC Research Institute Tech Rep. 95-10, 1995.

[2] R. Ohbuchi, H. Masuda, and M. Aono, "Watermarking ThreeDimensional Polygonal Models Through Geometric and Topological Modification," IEEE JSAC, pp. 551-560, 1998.

[3] X. Mao, M. Shiba, and A. Imamiya, "Watermarking 3D Geometric Models Through Triangle Subdivision," Proc. of Security and Watermarking of Multimedia Contents III,IS\&T/SPIES's Electronic Imaging, pp. 253-260, Jan. 2001.

[4] O. Benedens, "Geometry-Based Watermarking of 3D Models," IEEE CG\&A, pp. 46-55, Jan. /Feb. 1999.

[5] S. Kanai, H. Date, and T. Kishinami, "Digital Watermarking for 3D Polygons using Multiresolution Wavelet Decomposition," Proc. Sixth IFIP WG 5.2 GEO-6, pp. 296-307, Dec. 1998. 